\title{
Reactivity of ozone with solid potassium iodide investigated by atomic force microscopy Matthew A. Brown, ${ }^{1}$ Paul D. Ashby, ${ }^{2}$ D. Frank Ogletree, ${ }^{2}$ Miquel Salmeron ${ }^{2}$ and John C. Hemminger ${ }^{1 *}$
}

\author{
1.Department of Chemistry and AirUCI, University of California, Irvine, California 92697 \\ 2. Molecular Foundry, Lawrence Berkeley National Laboratory, University of California, Berkeley, California 94720
}

RECEIVED DATE (automatically inserted by publisher); jchemmin@uci.edu

\begin{abstract}
The reaction of ozone with the (100) plane of solid potassium iodide (KI) was investigated using atomic force microscopy (AFM). The reaction forming potassium iodate $\left(\mathrm{KIO}_{3}\right)$ initiates at step edges prior to reacting on the flat terraces. Small domains of $\mathrm{KIO}_{3}$, initially $3.8 \AA$ in height are formed on the top of step edges. Following reaction at the step edge, domains of $\mathrm{KIO}_{3}$ are formed across the terraces. With prolonged exposure to ozone, $\mathrm{KIO}_{3}$ domains nucleate further growth until the surface is evenly covered with $\mathrm{KIO}_{3}$ particles that are $4-6 \mathrm{~nm}$ in height, at which point the surface is passivated and the reaction terminates.
\end{abstract}

Introduction. Gas-phase halogens and halogenated oxides (X, XO, $\mathrm{X}_{2}, \mathrm{XY}, \mathrm{OXO}, \mathrm{HOX}, \mathrm{XONO}_{2}, \mathrm{XNO}_{2}$, where $\mathrm{X}, \mathrm{Y}=\mathrm{Cl}$, $\mathrm{Br}, \mathrm{I})$ affect the composition of the atmosphere as they play prominent roles in key events such as the oxidative capacity of the atmosphere, the global ozone budget, and aerosol nucleation and growth. ${ }^{1,2}$ The fundamental mechanisms responsible for gas-phase halogen release in the atmosphere are not well understood. Sea-salt aerosols have been shown to readily react with oxidants such as ozone and the subsequent reactions are suggested as a possible mechanism for the introduction of gas-phase reactive molecular halogens into the troposphere. ${ }^{3}$

Sea-salt droplets are present in the troposphere as aerosols, generated by wave-action in environments where the relative humidity exceeds that of their deliquescence point. In inland environments sea-salt particles are also found in the troposphere where they remain covered in thin water films. In addition, sea-salt ice is important in the polar-regions. ${ }^{4}$

Experimental studies have shown that in bromide-doped $\mathrm{NaCl}$ single crystals, at doping ratios as low as $0.001 \mathrm{~mol} \%$, $\mathrm{Br}^{-}$is greatly enhanced at the surface following subsequent drying of the deliquesced particle. ${ }^{5,6}$ As such, sea-salt aerosols that are transported to inland environments of low relative humidity are expected to retain the interfacial enhancement of the larger more polarizable anions that have been predicted theoretically and confirmed experimentally in aqueous solutions..$^{7-9}$ The surfaces of solid crystalline sea-salt particles are similarly expected to have a much higher concentration of iodide than in the bulk.

Previous x-ray photoelectron spectroscopy (XPS) studies have shown, under conditions in which a low defect density KI surface is completely devoid of adsorbed water molecules, that surface oxidation by ozone results in a self-passivating layer of $\mathrm{KIO}_{3} \cdot{ }^{10}$ XPS provides chemical identification of the reacting surface and products but is unable to provide spatial characterization of the reacting interface. Fluidized-bed reactor studies have shown the uptake of ozone to scale with iodide surface area, consistent with surface passivation. ${ }^{11}$

AFM is a non-destructive local probe technique that provides topographical and chemical information about the surface with atomic resolution. AFM imaging complements the previous XPS studies and provides a detailed microscopic understanding of the reacting interface. Herein, we present two fundamental results from the model system, $\mathrm{KI}_{(\mathrm{s})}+3 \mathrm{O}_{3(\mathrm{~g})}$ $\rightarrow \mathrm{KIO}_{3(\mathrm{~s})}+3 \mathrm{O}_{2(\mathrm{~g})}$. First, the oxidation of dry $\mathrm{KI}$ to form $\mathrm{KIO}_{3}$ initiates at step edges before reacting across sample terraces. Second, a passivating layer is formed when small particles of $\mathrm{KIO}_{3}$, typically $10-30 \mathrm{~nm}$ in width and 4-6 nm in height are tightly packed across the sample surface creating a surface with a RMS roughness of $3.2 \mathrm{~nm}$.

Experimental. The experimental details of the XPS studies (carried out at beamline 9.3.2 of the Advanced Light Source) have been described elsewhere. ${ }^{10,12}$ Solid samples of KI (Hilger
Crystal, UK) were cleaved along the (100) plane and immediately transferred to vacuum. Ozone was dosed through a thin Teflon tube that was placed directly in front of the sample surface. After each ozone dose, XPS peak areas for $\mathrm{I}(4 \mathrm{~d})$ and $\mathrm{K}(2 \mathrm{p})$ were measured. The photoemission peaks of $\mathrm{I}^{-}$ (4d) (un-reacted iodide) and $\mathrm{I}^{+5}(4 \mathrm{~d})$ (oxidized iodate) are well separated in the spectra allowing both species to be quantified. The photoemission spectra showed no evidence of other iodine oxidation states.

Topographic images were collected on a commercial Asylum Research MFP3D instrument in the attractive noncontact regime of $\mathrm{AC}$ mode, where the oscillating cantilever maintains feedback on attractive tip-sample interactions facilitating non-destructive imaging of the KI surface. Highly doped silicon tips (Budget Sensors) with a force constant of 3 $\mathrm{N} / \mathrm{m}$ were used throughout. KI samples were prepared by cleavage along the (100) plane and transferred to a sealed Teflon gas cell containing a humidity sensor, a modified version of the commercial liquid cell. Water vapor was introduced through a small Teflon tube inserted into the sample cell to mitigate the surface static charge generated during sample cleavage. After discharging and subsequent drying of the surface, ozone was introduced to the cell. All AFM measurements and ozone exposures were carried out in a dry environment where the relative humidity was below $4 \%$ at a total cell pressure of 1 atmosphere and ambient temperature.
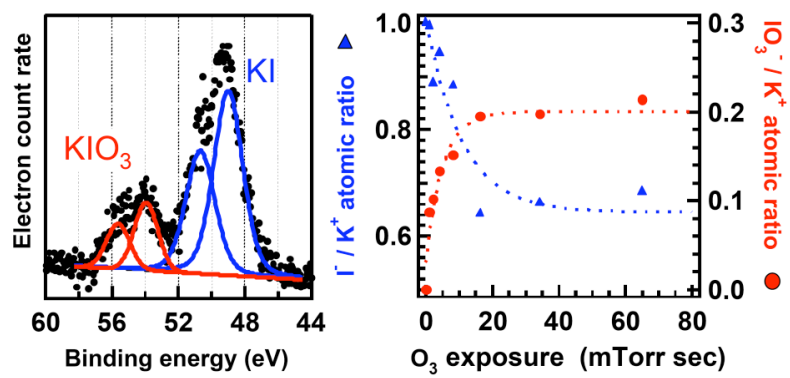

Figure 1: XPS results show the presence of two well-defined I(4d) doublets (left panel) separated by $4.9 \mathrm{eV}$. The XP spectrum shown at the left was obtained following an ozone exposure of 16 mTorr seconds. The doublet at low binding energy is assigned to the iodide of the KI starting material, while the doublet at high binding energy is the oxidized iodate. Uptake measurements (right panel) show that the oxidation process saturates following prolonged exposures to ozone.

Results. XPS was used to characterize and quantify the reaction to form $\mathrm{KIO}_{3}$ from $\mathrm{KI}$ as the exposure to ozone was increased. Figure 1 (left panel) shows a XP spectra of the I(4d) region following surface passivation due to iodate formation. Two clear doublets separated by $4.9 \mathrm{eV}$ are present. The doublet at low binding energy is assigned to the iodide (-1 oxidation state) of the KI starting material, while the higher binding energy doublet is assigned to the oxidized iodate $(+5$ oxidation state). Uptake measurements as a function of increasing ozone exposure are shown in Figure 1 (right panel). 
Initially there is no $\mathrm{KIO}_{3}$ present on the surface and a stoichiometric iodide to potassium ratio is measured. As the sample is exposed to increasing amounts of ozone there is a decrease in the iodide to potassium ratio and an increase in the iodate to potassium ratio. The surface concentration of $\mathrm{KIO}_{3}$ saturates following prolonged exposures to ozone. The average reactive sticking coefficient is $1.4( \pm 0.7) \times 10^{-4} \cdot{ }^{10}$

AFM images presented in Figure 2 show the initial reaction to form $\mathrm{KIO}_{3}$ at the KI surface. The original KI (100) plane (following a brief exposure to water vapor) is shown in Figure 2A. The surface has a RMS roughness of $0.3 \AA$, due mostly to instrument error. As the relative humidity is increased, surface ions become solvated and mobile, facilitating redistribution across the cleaved plane in order to dissipate surface static charge that is generated during the cleavage process. Rounding of the step edges results from a reduction in the surface energy. ${ }^{13}$ The onset of reactive uptake with ozone is shown in Figure 2B. The reaction initially proceeds along step edges, decorating the length of the edge with small particles of $\mathrm{KIO}_{3}$ that are $\sim 3.8 \AA$ in height, consistent with a single iodate sitting on the surface. Following reactivity of the step edge sites, the oxidation then takes place across the sample terraces as shown in Figure 3A. Small domains of potassium iodate nucleate before growing into nanometer sized particles of potassium iodate as the surface is exposed to increasing amounts of ozone (Figure 3B).

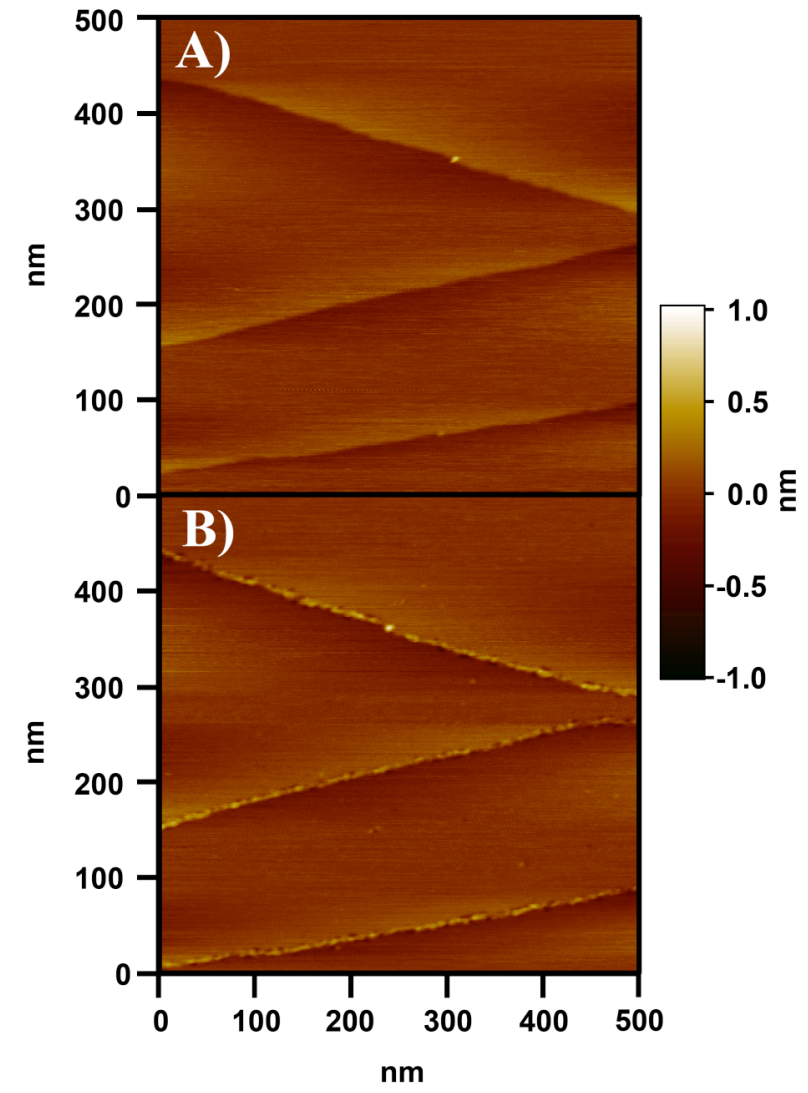

Figure 2: Topographic images of the KI surface during initial oxidation to form $\mathrm{KIO}_{3}$. The KI surface with monotonic step edges $\sim 3.5 \AA$ in height is shown in $(A)$. As the reaction begins, the step edges become decorated with small particles of $\mathrm{KIO}_{3}(B)$.

Initial oxidation occurs along the step edge and material migrates to form small particles along the edge. Figure 4A shows small particles of $\mathrm{KIO}_{3}$ that have decorated a step edge of the KI (100) surface. The corresponding section through a single particle is shown in Figure 4C. The section reveals that there is mass transport of material from the KI substrate during island growth, leaving pits adjacent to the islands. As the reaction proceeds further, domains of $\mathrm{KIO}_{3}$ are generated on the flat terraces of the sample. As these domains, which serve as nucleation sites, grow into particles with increasing ozone exposure, similar substrate pits are formed. Figure 4B shows a small particle of $\mathrm{KIO}_{3}, 1.5 \mathrm{~nm}$ in height on a flat terrace. The corresponding section is shown in Figure 4D. Erosion of the underlying KI surface has resulted in a pit from which the material has been transferred to the newly formed $\mathrm{KIO}_{3}$ particle.

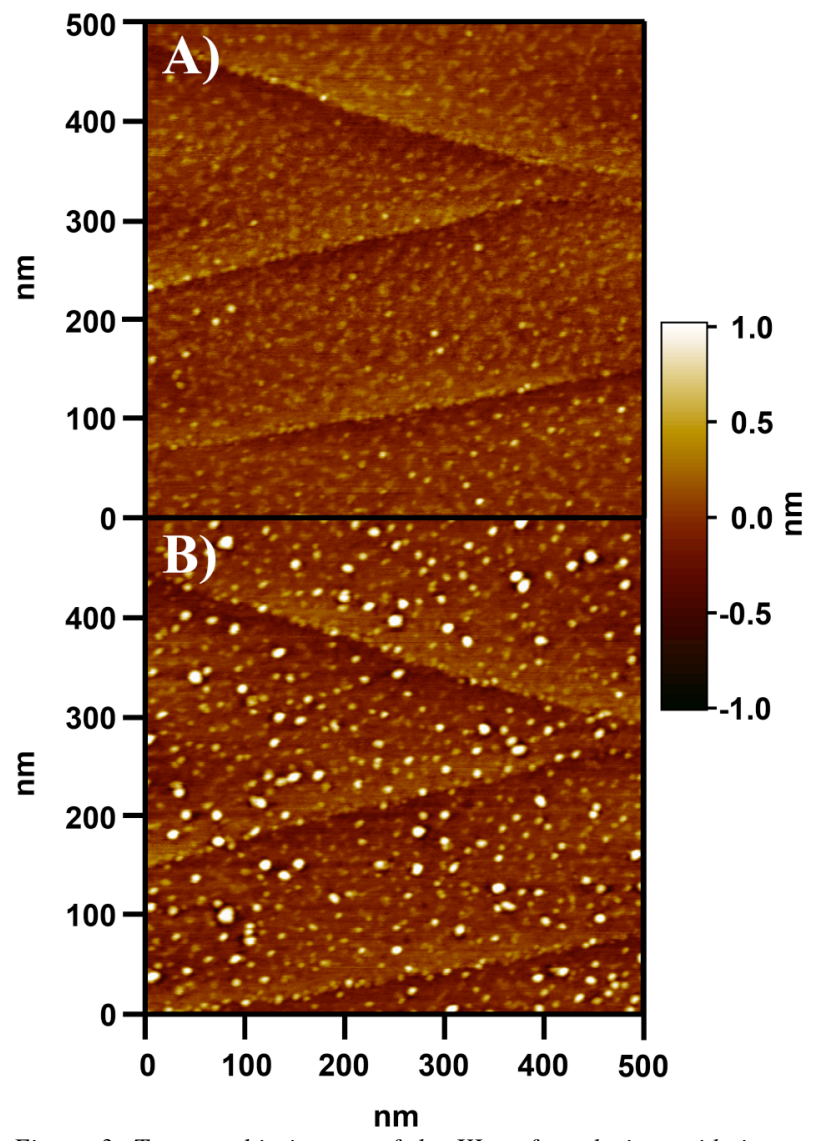

Figure 3: Topographic images of the KI surface during oxidation to form $\mathrm{KIO}_{3}$. With increasing ozone exposure, oxidation occurs across the sample terraces, nucleating additional islands of $\mathrm{KIO}_{3}(\mathrm{~A})$, which grow with increasing exposure to ozone $(B)$.

Figure 5 shows the KI surface following extended exposure to ozone in which the surface is saturated in $\mathrm{KIO}_{3}$. The small domains of $\mathrm{KIO}_{3}$ initially decorating the step edges and across the flat terraces seen in Figures 2 and 3 have grown in size and are now 10-30 $\mathrm{nm}$ in width, 4-6 $\mathrm{nm}$ in height and are densely packed across the entire sample surface. No further evolution of the island structure was observed in dry environments. When the oxidized KI surface was imaged in AFM contact mode, the iodate islands were easily displaced by the scanning tip, consistent with a weak interaction between the particle and the substrate due to an island-substrate lattice mismatch and associated strain.

Oxidation of the KI (100) surface by ozone to form $\mathrm{KIO}_{3}$ does not take place as a simple layer-by-layer process. We believe the passivating layer is comprised of small particles of $\mathrm{KIO}_{3}$, residing on top of the underlying KI surface. Additional electrostatic (Kelvin probe) AFM experiments, which we do not describe here in detail, were carried out on a partially reacted but not yet passivated surface. These experiments show a difference in contact potential between islands and the flat unreacted terraces, consistent with a difference in composition. Also, the XPS study suggests that the flat terrace regions could have at most 1 or 2 iodate layers of coverage. ${ }^{10}$ 
Prior to ozone exposure the cleaved (100) plane of KI is flat within the instrumental noise, while the reacted $\mathrm{KIO}_{3}$ surface has a RMS roughness of $3.2 \mathrm{~nm}$.
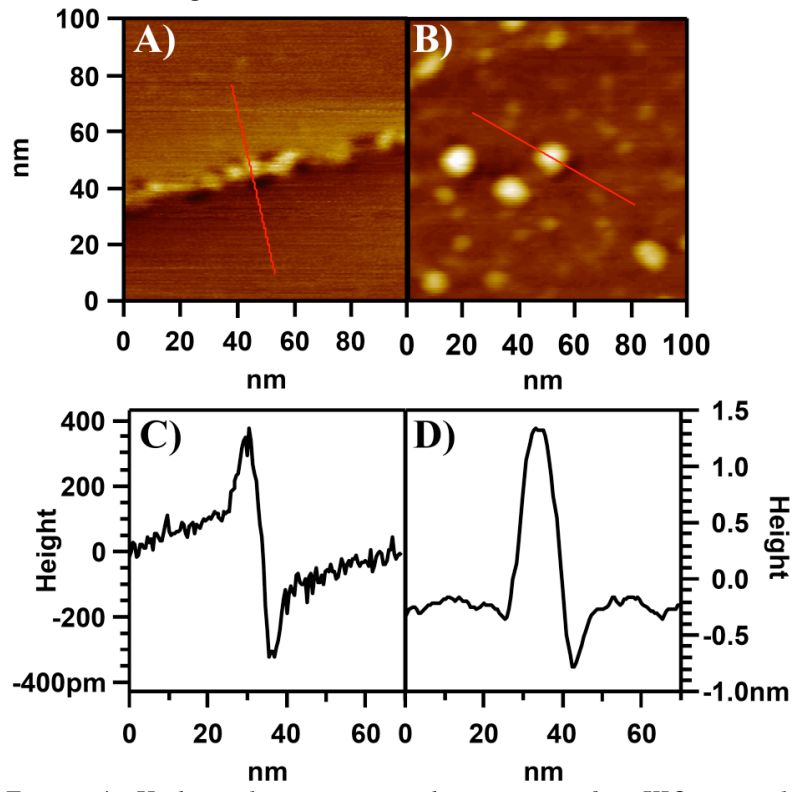

Figure 4: High-resolution topographic images of a $\mathrm{KIO}_{3}$ particle decorating the step edge site (A) (1 nm scale) and on a terrace (B) (3 $n m$ scale). Sections through the particles are shown in $(C)$ and $(D)$, respectively. Initial oxidation selectively takes place along the step edge with material being transferred from the substrate to the step edge site $(C)$. Similar mass transport is observed on the terraces $(D)$. Due to the finite size of the AFM tip, the widths of the particles are overestimated, and the sizes of the holes are underestimated in the topographic images.

Discussion. The reaction of ozone with solid KI to form $\mathrm{KIO}_{3}$ is thermodynamically favored according to the reaction

$$
\mathrm{KI}_{(\mathrm{s})}+3 \mathrm{O}_{3(\mathrm{~g})} \rightarrow \mathrm{KIO}_{3(\mathrm{~s})}+3 \mathrm{O}_{2(\mathrm{~g})}
$$

with a $\Delta \mathrm{G}_{\mathrm{rxn}}^{\mathrm{o}}=-579 \mathrm{KJ} / \mathrm{mol}^{14}{ }^{14}$ The continued reaction to form periodate $\left(\mathrm{IO}_{4}^{-}\right)$is further energetically favored with a $\Delta \mathrm{G}_{\mathrm{rxn}}^{\mathrm{o}}=$ $-685 \mathrm{KJ} / \mathrm{mol}^{14}{ }^{14}$ There is however, no evidence for the formation of periodate in our experiments. The photoelectron peaks of $\mathrm{I}(4 \mathrm{~d})$ in periodate $\left(\mathrm{I}^{+7}\right)$ are known to be shifted to higher binding energy by $6.0 \mathrm{eV}$ relative to the $\mathrm{I}^{-}$of the $\mathrm{KI}$ starting material, $1.1 \mathrm{eV}$ higher then the shift observed for the $\mathrm{I}^{+5}$ of the iodate. ${ }^{15}$ The periodate would therefore be easily detected in the XPS experiments if it were formed. In solution, iodide is known to rapidly consume ozone, and is thought to form hypoiodide $\left(\mathrm{IO}^{-}\right){ }^{16-18}$ There is no spectroscopic evidence of stable intermediates such as $\mathrm{IO}^{-}$or $\mathrm{IO}_{2}^{-}$in the formation of the final $\mathrm{IO}_{3}^{-}$product that we observe, although they cannot be ruled out as short-lived intermediates.

The KI crystal has a rock salt structure with a lattice constant of $7.07 \AA^{19}$ while the $\mathrm{KIO}_{3}$ crystal has a triclinic structure with lattice constants of $7.74,7.72$ and $7.73 \AA{ }^{20}$ making the two crystal structures incommensurate. As the reaction proceeds to form $\mathrm{KIO}_{3}$, the incompatibility of the two crystal structures causes structural rearrangement as a means to relieve stress. The rearrangement of the underlying structure causes mass transfer to the $\mathrm{KIO}_{3}$ particles generating pits that form adjacent to the new particles (seen in Figure 4).

Initial oxidation of the dry KI surface takes place along the step edge sites. Structural rearrangement of the KI surface upon oxidation at the step edge has lower activation energy than on the flat terraces. The lack of material at the step edge may reduce the stress involved in accommodating the larger anion (iodate). Adjacent oxidized material transfers to the step to form small homogeneous domains of $\mathrm{KIO}_{3}$. Following initial oxidation along the step edges the reaction proceeds on the flat terraces. Initial nucleation domains of $\mathrm{KIO}_{3}$ are formed across the sample terraces (Figure $3 \mathrm{~A}$ ) that are $\sim 3.8 \AA$ in height. It is difficult to put accurate lateral dimensions to these initial domains due to convolution with the tip shape, but they are generally smaller than $5 \mathrm{~nm}$ in width. As the reaction continues these initial domains serve as nucleation sites that evolve into larger particles of $\mathrm{KIO}_{3}$ (Figure 3B). Careful analysis reveals that few new domains of $\mathrm{KIO}_{3}$ develop between Figures $3 \mathrm{~A}$ and $3 \mathrm{~B}$. Once domains of $\mathrm{KIO}_{3}$ fill the initial reaction sites, they evolve into larger particles with increased ozone exposure. Although the reaction to form $\mathrm{KIO}_{3}$ initially takes place along the step edges, additional domains nucleate across the terraces before the initial particles along the step edge grow to their final size. The domains of $\mathrm{KIO}_{3}$ across the surface, both along the step edges and terraces, continue to evolve until surface passivation occurs. The passivating layer consists of small $\mathrm{KIO}_{3}$ particles $10-30 \mathrm{~nm}$ in width and 4-6 nm in height.

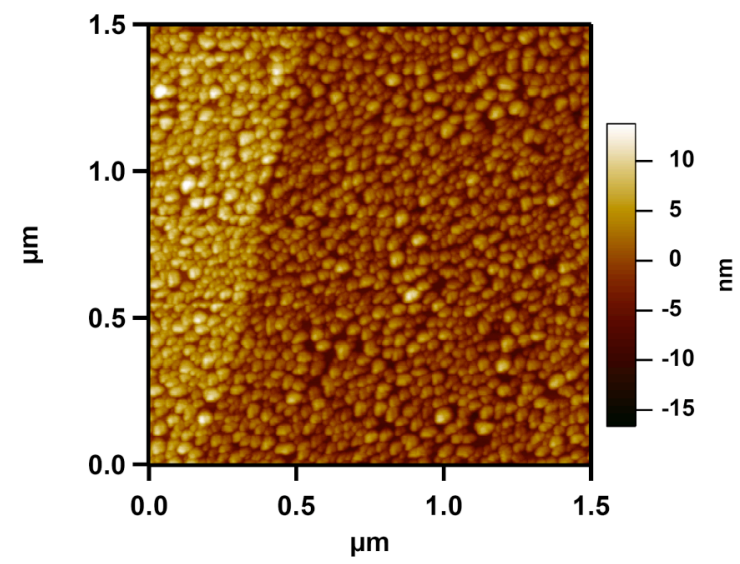

Figure 5: Topographic AC-mode AFM image of the KI (100) surface following a prolonged exposure to ozone in which the surface is covered by a passivating layer of $\mathrm{KIO}_{3}$. The passivating layer consists of small particles of $\mathrm{KIO}_{3}, 4-6 \mathrm{~nm}$ in height and evenly distributed across the sample surface.

Conclusion. AFM studies of the reaction $\mathrm{KI}_{(\mathrm{s})}+3 \mathrm{O}_{3(\mathrm{~g})} \rightarrow$ $\mathrm{KIO}_{3(\mathrm{~s})}+3 \mathrm{O}_{2(\mathrm{~g})}$ have provided microscopic detail into the structure of the reacting surface. Oxidation forming $\mathrm{KIO}_{3}$ was shown to initiate at step edges along the sample surface. Step edge sites provide a locale where the KI substrate can more easily strain to accommodate the lattice mismatch of the newly oxidized material. Following initial oxidation of the step edge sites the reaction proceeds across the flat terraces. Small nucleation domains are first generated that continue to grow with increasing ozone exposure. With extended exposures to ozone the surface of KI (100) becomes passivated by $\mathrm{KIO}_{3}$. The layer of $\mathrm{KIO}_{3}$ consists of small particles, typically 10-30 $\mathrm{nm}$ in width and 4-6 $\mathrm{nm}$ in heights that are densely packed across both the sample step edges and terraces. It is evident from the results present herein that the resulting $\mathrm{KIO}_{3}$ product does not grow in a simple layer-by-layer method. Simple planar surface models are routinely used for the analysis of surface reaction kinetics experiments in laboratory studies of atmospheric reactions. However, the results presented here demonstrate that simple kinetic models that assume atomically smooth Frank-van der Merwe (FM) growth of reaction products do not accurately account for the topography of this reacting system. Kinetic models of heterogeneous atmospheric chemistry need to be developed that take into account the complexity of such interfacial processes. 
Acknowledgement. The AirUCI Environmental Molecular Sciences Institute under grant number CHE 0431312 from the National Science Foundation supported this work. The ALS and the ambient pressure $\mathrm{x}$-ray photoemission endstation at beamline 9.3.2 are supported by the Director, Office of Science, Office of Basic Energy Sciences, Division of Chemical Sciences, Geosciences, and Biosciences and Materials Sciences Divisions of the U.S. Department of Energy at the Lawrence Berkeley National Laboratory under contract DE-AC03-76SF00098. M.A.B. acknowledges the ALS for support through the Doctoral Fellowship program. Portions of this work were performed at the Molecular Foundry, Lawrence Berkeley National Laboratory, which is supported by the Office of Science, Office of Basic Energy Sciences, of the U.S. Department of Energy under contract No. DE-AC02$05 \mathrm{CH} 11231$.

\section{$\overline{R e f e r e n c e s}$}

1. Finlayson-Pitts, B. J.; Pitts Jr., J. N., Chemistry of the Upper and Lower Atmosphere. Academic Press: New York, 2000.

2. Seinfeld, J. H.; Pandis, S. N., Atmospheric chemistry and physics : from air pollution to climate change. Wiley: New York, 1998.

3. Enami, S.; Vecitis, C. D.; Cheng, J.; Hoffmann, M. R.; Colussi, A. J., Global inorganic source of atmospheric bromine. Journal of Physical Chemistry A 2007, 111, (36), 8749-8752.

4. Barrie, L.; Platt, U., Arctic tropospheric chemistry: an overview. Tellus Series B-Chemical and Physical Meteorology 1997, 49, (5), 450-454.

5. Ghosal, S.; Shbeeb, A.; Hemminger, J. C., Surface segregation of bromine in bromide doped $\mathrm{NaCl}$ : Implications for the seasonal variations in Arctic ozone. Geophysical Research Letters 2000, 27, (13), 1879-1882.

6. Ghosal, S.; Verdaguer, A.; Hemminger, J. C.; Salmeron, M., In situ study of water-induced segregation of bromide in bromide-doped sodium chloride by scanning polarization force microscopy. Journal of Physical Chemistry A 2005, 109, (21), 4744-4749.

7. Jungwirth, P.; Tobias, D. J., Specific ion effects at the air/water interface. Chemical Reviews 2006, 106, (4), 1259-1281.

8. Petersen, P. B.; Saykally, R. J., Confirmation of enhanced anion concentration at the liquid water surface. Chemical Physics Letters 2004, 397, (1-3), 51-55.

9. Ghosal, S.; Hemminger, J. C.; Bluhm, H.; Mun, B. S.; Hebenstreit, E. L. D.; Ketteler, G.; Ogletree, D. F.; Requejo, F. G.; Salmeron, M., Electron spectroscopy of aqueous solution interfaces reveals surface enhancement of halides. Science 2005, 307, (5709), 563-566.

10. Brown, M. A.; Newberg, J. T.; Krisch, M. J.; Mun, B. S.; Hemminger, J. C., Reactivity of ozone on solid potassium iodide. Journal of Physical Chemistry C 2007, 112, (14), 5520-5525.

11. Alebic-Juretic, A.; Cvitas, T.; Klasinc, L., Kinetics of heterogeneous ozone reactions. Chemosphere 2000, 41, (5), 667-670.

12. Newberg, J. T. XPS studies of alkali halide oxides, ozone exposure to alkali halides and halate-halide reactions in thin-film water. $\mathrm{PhD}$. Thesis, Department of Chemistry, University of California, Irvine, Irvine, 2005

13. Luna, M.; Rieutord, F.; Melman, N. A.; Dai, Q.; Salmeron, M., Adsorption of water on alkali halide surfaces studied by scanning polarization force microscopy. Journal of Physical Chemistry A 1998, 102, (34), 6793-6800.

14. Lide, D. R., Handbook of Chemistry and Physics. CRC Press: Cleveland, OH, 2004.

15. Fadley, C. S.; Hagstrom, S. B.; Klein, M. P.; Shirley, D. A., Chemical Effects on Core-Electron Binding Energies in Iodine and Europium. Journal of Chemical Physics 1968, 48, (8), 3779-3794.

16. Liu, Q.; Schurter, L. M.; Muller, C. E.; Aloisio, S.; Francisco, J. S.; Margerum, D. W., Kinetics and mechanisms of aqueous ozone reactions with bromide, sulfite, hydrogen sulfite, iodide, and nitrite ions. Inorganic Chemistry 2001, 40, (17), 4436-4442.

17. Garland, J. A.; Elzerman, A. W.; Penkett, S. A., The Mechanism for Dry Deposition of Ozone to Seawater Surfaces. Journal of Geophysical Research-Oceans and Atmospheres 1980, 85, (Nc12), 7488-7492.
18. Hoigne, J.; Bader, H.; Haag, W. R.; Staehelin, J., Rate Constants of Reactions of Ozone with Organic and Inorganic-Compounds in Water .3. Inorganic-Compounds and Radicals. Water Research 1985, 19, (8), 993-1004.

19. Hambling, P. G., The lattice constants and expansion coefficients of some halides. Acta Crystallographica 1953, 6, 98.

20. Lucas, B. W., Structure (neutron) of room temperature phase III potassium iodate. Acta Crystallographica Section C-Crystal Structure Communications 1984, 40, 1989-1992. 
TABLE OF CONTENTS GRAPHIC

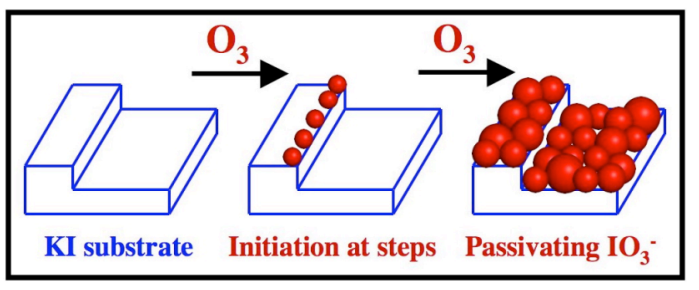

